\title{
El papel del FMI en la protección social: prometedores cambios en políticas y desafíos persistentes
}

\section{The IMF's role in social protection: Promising policy shifts and persisting challenges}

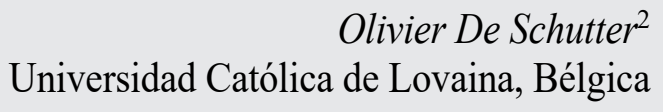

Nathalie Dubler ${ }^{3}$

Instituto de Estudios Políticos de París (Sciences Po), Francia

Paula Fernandez-Wulff

Universidad de Nueva York, Estados Unidos

Anabella Sosa ${ }^{5}$

Instituto de Estudios Políticos de París (Sciences Po), Francia

Revista Derechos en Acción ISSN 2525-1678/ e-ISSN 2525-1686

Año 6/№ 18 Verano 2020-2021 (21 diciembre a 20 marzo), 382-404

DOI: https://doi.org/10.24215/25251678e486

Resumen: Se puede afirmar que el Fondo Monetario Internacional (FMI) ha cambiado su postura sobre la protección social, entendida como el conjunto de medidas públicas destinadas a proteger a la población de los peligros sociales y económicos en caso de reducción o pérdida de ingresos. Desde los más altos cargos del FMI, se ha afirmado que se

\footnotetext{
1 Traducción al español de Natalia Barry.

2 Profesor, Université Catholique de Louvain; relator especial de la ONU sobre la extrema pobreza y los derechos humanos.

3 M.A., Paris School of International Affairs, Sciences Po.

4 Investigadora en Residencia, Center for Human Rights and Global Justice, New York University (NYU) School of Law; asesora senior del relator especial de la ONU sobre la extrema pobreza y los derechos humanos.

5 M.A., Paris School of International Affairs, Sciences Po. Las opiniones aquí vertidas no reflejan necesariamente las de las Naciones Unidas.
} 
produjo un cambio en el foco de su atención: de una concentración excesiva en ajustes estructurales, con frecuencia muy criticada, a un "nuevo" abordaje e interés en las cuestiones sociales a través del prisma de la "macrocrítica". En el presente artículo, se estudia la evolución y el alcance de este aparente cambio, dada la importancia del papel del FMI en la protección social: las recomendaciones macroeconómicas del organismo tienen una influencia decisiva sobre las decisiones de políticas a nivel nacional en relación con la protección social, mediante mecanismos como las recomendaciones y experiencia de vigilancia, las condicionalidades sobre préstamos y el papel del FMI como facilitador ante préstamos a terceros. También se analiza la respuesta del FMI a la crisis económica originada por la pandemia de la COVID-19 a fin de evaluar hasta qué punto se han modificado sus políticas en la realidad. Mostramos que, si bien se han concretado algunos cambios prometedores en las políticas — entre ellas, la reducción y hasta eliminación de algunas condicionalidades y la promoción de inversiones en el sector salud - la insistencia en el uso de medidas selectivas (targenting) para combatir la pobreza, en lugar del fomento de pisos de protección social universales, así como el enfoque persistente en la sustentabilidad fiscal, le han llevado a tener un enfoque ambiguo que menoscaba los avances en pos de hacer de la protección social un recurso universal, tal y como se comprometieron los gobiernos en la Recomendación 202 de la OIT (2012). Prevalece un marcado contraste entre, de un lado, los esfuerzos desplegados para satisfacer las necesidades de financiamiento a nivel de país, y del otro, la imposición de condicionalidades en nombre de la sustentabilidad fiscal. El FMI debe adoptar un enfoque en cuanto a la protección social basado en los derechos humanos, que supere su tradicional insistencia en medidas selectivas en pos de la universalidad, de manera que se logre avanzar más allá de la mitigación de los efectos de sus programas sobre sectores vulnerables. Un primer paso en esta dirección sería incrementar la cooperación con otras organizaciones internacionales, no solo el Banco Mundial, sino también UNICEF y la OIT, así como incorporarse a los esfuerzos existentes para el establecimiento de un Fondo Global para la Protección Social.

Abstract: The International Monetary Fund (IMF) has arguably shifted its position towards social protection, understood as a set of public measures intended to protect individuals from social and economic distress in 
cases of income reduction or loss. Leadership in the IMF has affirmed that there has been a change from its much-criticized focus on structural adjustments and austerity, to a "new" approach and interest in social issues through the lens of "macro-criticality." In this article, we survey the evolution and extent of this possible shift, given the important role of the IMF in social protection: the macro-economic recommendations the organization makes have a clear influence over countries' policy decisions related to social protection, through mechanisms including surveillance advice and expertise, conditionalities on lending, and its gatekeeping role for third-party lending. We examine the IMF's response to the economic crisis triggered by the COVID-19 pandemic to assess the extent to which its policies have truly shifted. We show that, while there have been promising policy changes, including lowering or even eliminating some conditionalities and promoting healthcare spending, its focus on targeted measures to combat poverty, as opposed to universal social protection floors, and its continued focus on fiscal sustainability, lead to an ambiguous approach that hinders progress to make social protection universal, as committed by governments in ILO Recommendation 202 (2012). We find that a sharp contrast remains between efforts deployed to meet countries' financing needs and the content of the conditionalities imposed in the name of fiscal sustainability. The IMF must adopt a human rights-based approach towards social protection, going beyond targeting and towards universality, and not simply mitigate the effects of its programs on the vulnerable. Increasing cooperation with other international organizations, not only the World Bank, but UNICEF and the ILO as well, and joining ongoing efforts for the establishment of a Global Fund for Social Protection, would be a first step in this direction

\section{Introducción}

Si bien la protección social ha ganado prominencia en la política internacional de los últimos años, ${ }^{6}$ los esfuerzos

\footnotetext{
6 Oficina de Evaluación Independiente del Fondo Monetario Internacional (2017). Informe de evaluación "The IMF and Social Protection". Disponible en inglés en: https://ieo.imf.org/ en/our-work/Evaluations/Completed/2017-0724-the-imf-and-social-protection.
} 
internacionales no logran aún convertirla en universal, ${ }^{7}$ tal como lo estipula la Recomendación 202 adoptada por la Conferencia Internacional del Trabajo en 2012. Será necesario el mejoramiento de la cooperación internacional si se desean alcanzar los ODS en 2030, y las organizaciones financieras internacionales tienen un importante papel que jugar en ese sentido.

El Fondo Monetario Internacional (FMI) es una de ellas, pero ¿qué papel puede desempeñar —si acaso desempeña alguno- en ese sentido? En abril de 2020, la directora del organismo, Kristalina Georgieva, declaró: "El FMI fue creado para tiempos como este: estamos aquí para desplegar la fuerza de la comunidad global, de manera que podamos ayudar a proteger a los más vulnerables y revitalizar la economía". ${ }^{8}$ En el informe "Perspectivas de la economía mundial 2020" (World Economic Outlook 2020) de octubre de 2020, el FMI subrayaba la necesidad de que los países incrementaran la inversión en el sector de la salud y, desde entonces, empezó a proporcionar apoyo internacional en forma de alivio de deudas y financiación en condiciones favorables mientras que, a la vez, insistía en la importancia del desarrollo de formas de tributación basadas en principios progresivos y en la cooperación internacional impositiva. ${ }^{9}$ Sin embargo, algunas organizaciones de la sociedad civil como el Comité para la Abolición de las Deudas Ilegítimas (CADTM, por su sigla en francés), argumentan que las medidas de austeridad del FMI debilitan los sistemas de salud nacionales y demoran una posible respuesta de políticas coordinadas, lo cual impide

\footnotetext{
7 Ver Isabel Ortiz, et al. (2017), "Fiscal Space for Social Protection and the SDGs: Options to Expand Social Investments in 187 Countries". Documento de trabajo sobre ESS de la OIT, núm. 48.

8 FMI, "Confronting the Crisis: Priorities for the Global Economy". Discurso de Kristalina Georgieva, (2020). Disponible en inglés en: https://www.imf.org/en/News/Articles/2020/04/07/ sp040920-SMs2020-Curtain-Raiser.

9 FMI, “World Economic Outlook 2020", (2020). Disponible en inglés y español en: https://www. imf.org/en/Publications/WEO/Issues/2020/09/30/world-economic-outlook-october-2020.
} 
que los países en desarrollo respondan adecuadamente a ciertas crisis sanitarias como la del ébola o, más recientemente, la de la COVID-19. Algunos observadores también han criticado fuertemente la falta de acciones concretas para honrar los compromisos asumidos por el Fondo al comienzo de la crisis. ${ }^{10}$

¿Cómo entender esas aparentes contradicciones? Por ser el principal organismo encargado de promover la estabilidad financiera y la cooperación monetaria internacionales, el FMI ejerce gran influencia sobre la protección social ${ }^{11}$ y su papel puede ser clave para asegurar que el gasto social nacional se oriente a políticas de protección social que no dejen a nadie atrás. De más está decir que el mandato del Fondo es asegurar la estabilidad financiera y, oficialmente, la protección social no está entre sus prioridades. Sin embargo, las prescripciones macroeconómicas del FMI pueden o no favorecer el que la protección social se entienda como prioridad en los países a los que asiste. Esto se debe a que el hecho de disponer de espacio fiscal y de poder preservar la credibilidad frente a los acreedores internacionales son factores determinantes en la toma de decisiones relacionadas con la protección social por parte de los gobiernos nacionales.

\section{Enfoque del FMI en cuanto a la protección social}

El mandato del FMI es asegurar la estabilidad del sistema monetario internacional y no contempla la protección social explícitamente. No obstante, ciertos ajustes estructurales y reformas económicas promovidos desde el FMI han influido directamente

\footnotetext{
10 Thomas Stubbs, William Kring, Christina Laskaridis, Alexander Kentikelenis, Kevin Gallagher, "Whatever it takes? The Global Financial Safety Net, COVID-19 and Developing Countries", en World Development, 2021, vol. 137. Disponible en inglés en: https://doi. org/10.1016/j.worlddev.2020.105171

11 Informe del Relator Especial sobre la extrema pobreza y los derechos humanos Philip Alston, "The International Monetary Fund and its Impact on Social Protection", (2018). Disponible en inglés en: https://srpoverty.org/wp-content/uploads/2018/08/international-monetary-fundimpact-on-social-protection-2018.pdf
} 
sobre el diseño y la implementación de la protección social en el mundo. En respuesta a las críticas recibidas por mantenerse al margen de esos impactos, con el tiempo, el FMI fue cambiando su postura frente a la protección social. ${ }^{12} \mathrm{Si}$ bien las políticas sociales no se abordaron de forma explícita hasta fines de 1990, la crisis financiera de 2008 ya hizo que el Fondo incluyera una referencia a los programas de seguridad social y a las condicionalidades de préstamos en los países menos desarrollados (PMD). ${ }^{13}$ Desde aquel momento, el Fondo comenzó a incluir condicionalidades no vinculantes que promovieran el gasto social, en especial en salud y educación. De hecho, un estudio de 2013 realizado por el mismo Fondo Monetario llegó a la conclusión de que "los programas apoyados por el FMI tienen un efecto positivo y significativo en el gasto social de los países de ingreso bajo". ${ }^{14}$ Un estudio publicado en 2017 por la Oficina de Evaluación Independiente (OEI) del Fondo señalaba que durante el período de 2006 a 2015, un promedio del $10 \%$ de los acuerdos promovidos por el FMI comprendían condicionalidades estructurales que se concentraban más directamente en la protección social o la fortalecían; ${ }^{15} \mathrm{y}$ agregaba que en los países de ingreso bajo, "el FMI a veces tenía una función significativa en la defensa del fortalecimiento de los programas de seguridad social". ${ }^{16}$

12 Oficina de Evaluación Independiente del Fondo Monetario Internacional, "The IMF and Social Protection", Documento de referencia Núm. BD/17-01/01, (2017).

13 Oficina de Evaluación Independiente del Fondo Monetario Internacional, "The IMF and Social Protection", Documento de referencia Núm. BD/17-01/01, (2017).

14 Benedict Clements, Sanjeev Gupta y Masahiro Nozaki, “What Happens to Social Spending in IMF-supported programmes?". Notas de discusión entre funcionarios del FMI (IMF Staff Discussion Note), (2011), 16. Disponible en inglés en: https://www.imf.org/external/pubs/ft/ sdn/2011/sdn1115.pdf

15 Oficina de Evaluación Independiente del Fondo Monetario Internacional, Informe de evaluación, "The IMF and Social Protection", (2017), 21. Disponible en inglés en: https://ieo. imf.org/en/our-work/Evaluations/Completed/2017-0724-the-imf-and-social-protection

16 Oficina de Evaluación Independiente del Fondo Monetario Internacional, Informe de evaluación, "The IMF and Social Protection", (2017), 22. Disponible en inglés en: https://ieo. imf.org/en/our-work/Evaluations/Completed/2017-0724-the-imf-and-social-protection 
El enfoque "nuevo" del FMI, promovido por primera vez durante la dirección de Christine Lagarde, expresaba un interés creciente en la pobreza, la desigualdad y las cuestiones sociales. ${ }^{17}$ En respuesta a las críticas y a la presión proveniente de la comunidad internacional, el Fondo cambió su discurso sobre protección social y pobreza en general y, a mediados de 2000, introdujo la noción de macrocrítica. ${ }^{18}$ Según el FMI, un problema se define como macrocrítico "si afecta o tiene el potencial de afectar la estabilidad interior o exterior". ${ }^{19}$ Un problema que el FMI habitualmente define como macrocrítico en la mayoría de los países es el gasto social y, dentro de este gasto, se halla la protección social. Con este modus operandi, el FMI analiza la macrocrítica del gasto social, poniendo el foco en la sustentabilidad fiscal y la adecuación del gasto, así como en la eficiencia al momento de destinar ese gasto. ${ }^{20} \mathrm{Sin}$ embargo, el Fondo todavía no dispone de un criterio estandarizado para determinar la macrocrítica: la decisión en cuanto a si son macrocríticas o no las intervenciones en un país en materia de protección social finalmente "quedan a criterio subjetivo del personal técnico del FMI". ${ }^{21}$

17 Barry Herman, "Social Protection Floors in the Financing for Development Agenda" Equipo de Tareas Interinstitucional sobre el Financiamiento para el Desarrollo (Inter-agency Task Force on Financing for Development), (2017). Serie documentos de trabajo.

18 Oficina de Evaluación Independiente del Fondo Monetario Internacional, Informe de evaluación "The IMF and Social Protection" (2017). Disponible en inglés en: https://ieo.imf. org/en/our-work/Evaluations/Completed/2017-0724-the-imf-and-social-protection

19 Documento normativo del FMI (IMF Policy Paper), "Una estrategia para la participación del FMI en el gasto social". Washington D.C., (2019). Disponible en inglés y español en: https://www.imf.org/en/Publications/Policy-Papers/Issues/2019/06/10/A-Strategy-for-IMFEngagement-on-Social-Spending-46975

20 Documento normativo del FMI (IMF Policy Paper), "Una estrategia para la participación del FMl en el gasto social". Washington D.C., (2019) Disponible en inglés y español en: https://www.imf.org/en/Publications/Policy-Papers/Issues/2019/06/10/A-Strategy-for-IMFEngagement-on-Social-Spending-46975

21 Clínica sobre organizaciones internacionales en la Facultad de Derecho de la Universidad de Nueva York (International Organizations Clinic at NYU School of Law), (2018), "IMF and Social Protection", 16-17, 48. En una declaración basada en las entrevistas al personal funcionario del 
La inclusión de políticas sociales dentro de los programas del Fondo se sustenta mayoritariamente en su colaboración con el Banco Mundial, entidad que lidera el diseño y la implementación de programas de seguridad social focalizados en la pobreza extrema. ${ }^{22}$ Existe una tradición consolidada en cuanto al apoyo que el Fondo obtiene de la experiencia del Banco Mundial en estos temas, y esto se refleja en las decisiones del Fondo sobre Políticas y Orientación desde la década de los años ochenta. En su informe "Financiamiento para el desarrollo" (Financing for Development) de 2015, el Fondo también destacaba la importancia de brindar a los países apoyo selectivo (targeted support) "a la vez de recalcar que la participación del Fondo en esas áreas debe centrarse en las políticas consideradas macrocríticas para conseguir un crecimiento económico sostenido y basarse en una colaboración estrecha con otras instituciones como el Banco Mundial". ${ }^{23}$

En línea con el enfoque del Banco Mundial, el FMI apoya la idea de reinventar la protección social de tal manera que ya no se busque generar trabajos para toda la vida con una cobertura social garantizada, sino un nuevo contrato social que introduzca un enfoque de universalismo progresivo junto con un uso innovador de la tecnología digital. Por ejemplo, en India, la Transferencia Directa de Beneficios ofrece subsidios disponibles directamente en las cuentas bancarias de los más pobres, lo cual reduce los costos administrativos y las potenciales demoras de los pagos. ${ }^{24}$ En 2018, el FMI otorgó a Argentina un

\footnotetext{
FMI se lee: "Un miembro del personal funcionario observó que prácticamente cualquier asunto de un país puede ser macrocrítico". Disponible en inglés en: https://www.iilj.org/wp-content/ uploads/2018/06/The-IMF-and-Social-Protection-NYU-IO-Clinic-Fall-2017.pdf

22 Oficina de Evaluación Independiente del Fondo Monetario Internacional, Informe de evaluación, "The IMF and Social Protection". Documento de referencia Núm. BD/17-01/01, (2017); Coalición Global por los Pisos de Protección Social, “The IMF's New Policy Framework on Social Protection, (2019), Disponible en inglés en: socialprotectionfloorscoalition. org/2019/01/the-imfs-new-policy-framework-on-social-protection/

23 FMI, "Financing for Development: Revisiting the Monterrey Consensus", (2015), 3. Disponible en inglés en: https://www.imf.org/external/np/pp/eng/2015/061515.pdf

24 Michal Rutkowski, "Reimagining Social Protection. New Systems that Do Not Rely on Standard Employment Contracts Are Needed", en: Revista del FMI sobre finanzas y desarrollo
} 
préstamo de 57000 millones de dólares —lo cual representaba el préstamo más grande de la historia del organismo- que contemplaba un gasto mínimo en asistencia social como criterio de eficacia. ${ }^{25}$

El interés y la participación del FMI en temas vinculados con el gasto social se ha incrementado en los últimos años. Las más recientes investigaciones del organismo en el tema apoyan la idea de que la reducción de la desigualdad promueve el crecimiento económico. ${ }^{26}$ En su informe publicado en 2014 basado en datos recogidos de Guatemala, Malawi y Myanmar, el FMI muestra que el crecimiento económico no siempre trae aparejado una reducción en la desigualdad y que, por el contrario, algunas medidas económicas que buscan fortalecer el crecimiento económico tienen por efecto secundario recrudecer esta desigualdad. ${ }^{27}$

Para contrarrestar esto, la propuesta del Fondo consiste en usar estrategias de mitigación, como las transferencias de efectivo selectivas, para impulsar la productividad de ciertas poblaciones específicas. ${ }^{28}$ Según el personal técnico del Fondo, estas medidas selectivas tienen "mayor impacto contra la pobreza,

(IMF Finance \& Development Magazine), 2018. Disponible en inglés en: https://www.imf.org/ external/pubs/ft/fandd/2018/12/pdf/world-bank-reimagining-social-protection-rutkowski.pdf

25 Coalición Global por los Pisos de Protección Social, "The IMF's New Policy Framework on Social Protection", (2019), Disponible en inglés en: http://www.socialprotectionfloorscoalition.org/2019/01/the-imfs-new-policy-framework-on-social-protection/

26 Andrew Berg, Jonathan D. Ostry y Charalambos G. Tsangarides, "Redistribution, Inequality, and Growth". Notas de discusión del personal funcionario del FMI (IMF Staff Discussion Note), (2017). Disponible en inglés en: https://www.imf.org/external/pubs/ft/sdn/2014/ sdn1402.pdf.

27 Andrew Berg y Charalambos G. Tsangarides, "Redistribution, Inequality, and Growth". Notas de discusión del personal funcionario del FMI (IMF Staff Discussion Note), (2017). Disponible en inglés en: https://www.imf.org/external/pubs/ft/sdn/2014/sdn1402.pdf.

28 Stefania Fabrizio, Roland Kpodar y Chris Lane, "El FMI Apoya los Objetivos de Desarrollo Sostenible de las Naciones Unidas". Blog del FMI, (2017). Disponible en inglés y español en: https://blogs.imf.org/2017/07/19/imf-support-for-the-united-nations-sustainable-development-goals/ 
considerado por cada transferencia por separado" 29 en los países de ingreso bajo (PIB) y, así, se considera que las políticas de protección social selectivas mejoran la eficiencia de los desembolsos y, en consecuencia, protegen mejor a los más vulnerables. ${ }^{30}$ Los programas de gasto social del Fondo, por lo tanto, se han concentrado principalmente en focalizarse en los sectores más pobres y vulnerables de la población. Esta asistencia se proporcionó principalmente en forma de ayuda directa a los sectores de ingreso bajo a través de programas de mínimos de seguridad social. ${ }^{31}$

El FMI insiste en que su priorización de intervenciones en favor del gasto social específicamente focalizado en los sectores más pobres es coherente con el hecho de que los países de ingreso bajo tienen limitada capacidad fiscal y recursos. ${ }^{32}$ Por lo tanto, el Fondo no parece compartir el enfoque universal de la protección social de la OIT que va en concordancia con los Objetivos de Desarrollo Sustentable 2030. ${ }^{33}$ Los detractores del Fondo han señalado que la entidad no pone suficiente énfasis en los efectos distribucionales de las políticas sociales que promueve, ni en los costos sociales que conlleva una cobertura

29 David Coady y Nghia-Piotr Le, Designing Fiscal Redistribution: The Role of Universal and Targeted Transfers. Documento de trabajo del FMI, (2020). Disponible en inglés en: https:// www.imf.org/en/Publications/WP/Issues/2020/06/26/Designing-Fiscal-RedistributionThe-Role-of-Universal-and-Targeted-Transfers-49502

30 Oficina de Evaluación Independiente del Fondo Monetario Internacional, Informe de evaluación, "The IMF and Social Protection", (2017). Disponible en inglés en: https://ieo. imf.org/en/our-work/Evaluations/Completed/2017-0724-the-imf-and-social-protection

31 Oficina de Evaluación Independiente del Fondo Monetario Internacional, Informe de evaluación, “The IMF and Social Protection", 11-12, (2017). Disponible en inglés en: https:// ieo.imf.org/en/our-work/Evaluations/Completed/2017-0724-the-imf-and-social-protection

32 Zaineb Majoka y Robert Palacios, Targeting versus Universality: Is There a Middle Ground? 22 Social Protection and Jobs Policy and Technical Note; World Bank, Washington, D.C. Disponible en inglés en: http://hdl.handle.net/10986/32789

33 Oficina de Evaluación Independiente del Fondo Monetario Internacional, Informe de evaluación, "The IMF and Social Protection", (2017). Disponible en inglés en: https://ieo. imf.org/en/our-work/Evaluations/Completed/2017-0724-the-imf-and-social-protection 
de protección social insuficiente. ${ }^{34}$ El ex Relator Especial de las Naciones Unidas sobre la pobreza extrema y los derechos humanos atribuye esto a la postura del FMI al hecho de que los derechos humanos no son relevantes per se para la política macroeconómica. ${ }^{35}$ Otros actores, como la Coalición Global por los Pisos de Protección Social, también destacan que el Fondo debería ir integrando progresivamente un enfoque más basado en los derechos humanos en lo referente a la protección social. ${ }^{36}$

\section{Mecanismos de influencia del FMI sobre las prioridades de políticas a nivel país}

El enfoque nuevo del FMI, en cuanto a considerar la protección social a la luz de la macrocrítica del gasto social, se ha visto atacado por más de un frente. ${ }^{37}$ Puede afirmarse que las condicionalidades de préstamos reducen el espacio fiscal disponible para el gasto social de un país. Los programas de ajustes estructurales del Fondo, si bien menos frecuentes en la actualidad, han reducido el espacio para las políticas y con ello el acceso a ciertas herramientas para esas políticas para luchar contra la pobreza. Por último, el énfasis en el gasto social selectivo puede dejar atrás a algunos sectores vulnerables de la población y, como se señaló, no es consistente con un enfoque de la protección

34 Oficina de Evaluación Independiente del Fondo Monetario Internacional, Informe de evaluación, "The IMF and Social Protection", (2017). Disponible en inglés en: https://ieo.imf. org/en/our-work/Evaluations/Completed/2017-0724-the-imf-and-social-protection

35 Informe del Relator Especial sobre la extrema pobreza y los derechos humanos Philip Alston, "The International Monetary Fund and its Impact on Social Protection", (2018). Disponible en inglés en: https://srpoverty.org/wp-content/uploads/2018/08/international-monetaryfund-impact-on-social-protection-2018.pdf

36 Coalición Global por los Pisos de Protección Social, "The IMF's New Policy Framework on Social Protection", (2019). Disponible en inglés en: socialprotectionfloorscoalition. org/2019/01/the-imfs-new-policy-framework-on-social-protection/.

37 Clínica sobre organizaciones internacionales en la Facultad de Derecho de la Universidad de Nueva York (International Organizations Clinic at NYU School of Law) (2018), "IMF and Social Protection"). Disponible en inglés en: https://www.iilj.org/wp-content/uploads/2018/06/ The-IMF-and-Social-Protection-NYU-IO-Clinic-Fall-2017.pdf. 
social universal basado en los derechos humanos. Estas críticas quedaron cristalizadas en un informe publicado por el personal técnico del FMI el 14 de septiembre de 2020, referente a su participación en el gasto social durante la pandemia de la COVID-19. En el documento, el FMI presenta un enfoque macrocrítico para fortalecer el gasto social y concuerda con la necesidad de un gasto social más adecuado, eficiente y sustentable, al hacer mención de la necesidad de un aumento progresivo (scale up) de la protección social a los sectores pobres y vulnerables. Durante la fase de contención de la crisis de la COVID-19, parecía que el FMI estaba abierto a negociar y tal vez a modificar su enfoque, pero a medida que se acercó la fase de recuperación, el Fondo pronto revirtió la tendencia y pasó a priorizar la selectividad y a tomar distancia con la universalización. ${ }^{38}$ Así, el informe omite considerar la pandemia como oportunidad única de repensar el enfoque de la protección social. ${ }^{39}$

Con el fin de evaluar el alcance de estas variadas críticas, resulta de utilidad recuperar las tres vías por las cuales el Fondo ejerce influencia decisiva sobre las decisiones a nivel país.

\section{Recomendaciones de vigilancia y experiencia}

Diferentes analistas han observado que "las recomendaciones de vigilancia del FMI se han convertido en la vía de influencia de la institución sobre las políticas de los Estados miembros". ${ }^{40}$

\footnotetext{
38 Irene Yackovlev, et al., "How to Operationalize IMF Engagement on Social Spending during and in the Aftermath of the COVID-19 Crisis", en How To Note 20/02 FMI, 14 de septiembre de 2020, 3. Disponible en inglés en: https://www.imf.org/en/ Publications/Fiscal-Affairs-Department-How-To-Notes/Issues/2020/09/14/How-to-Operationalize-IMF-Engagement-on-Social-Spending-during-and-in-the-aftermath-of-the-49718

39 Irene Yackovlev, et al., "How to Operationalize IMF Engagement on Social Spending during and in the Aftermath of the COVID-19 Crisis", en How To Note 20/02 FMI, 14 de septiembre de 2020. Disponible en inglés en: https://www.imf.org/en/ Publications/Fiscal-Affairs-Department-How-To-Notes/Issues/2020/09/14/How-to-Operationalize-IMF-Engagement-on-Social-Spending-during-and-in-the-aftermath-of-the-49718

40 Clínica sobre organizaciones internacionales en la Facultad de Derecho de la Universidad de Nueva York (International Organizations Clinic at NYU School of Law) (2018), “IMF and Social
} 
La experiencia del FMI en cuanto a vigilancia e investigación tiene una fuerte influencia en los sistemas de protección social de cada país. Las actividades vinculadas a la vigilancia permiten al FMI establecer planes a largo plazo para los países miembros e influir en ellos.

En los últimos años, el Fondo ha mostrado un interés particular por los Estados "frágiles", donde las medidas de austeridad se prescriben como paso clave hacia el éxito del país. ${ }^{41}$ Un informe elaborado por la OIT y otras entidades asociadas, publicado en 2015, también encontró que la vigilancia del FMI ha conducido a los países en vías de desarrollo a efectuar recortes importantes en los fondos destinados al gasto público, lo cual impacta directamente en la seguridad social de la población. ${ }^{42}$

\section{Condicionalidades sobre préstamos}

La segunda vía por la cual el Fondo ejerce influencia sobre las políticas de un país es mediante el establecimiento de condicionalidades sobre los préstamos. Estas condicionalidades pueden conllevar la reducción del déficit público mediante el recorte del gasto social o la privatización de empresas estatales, con el objetivo general de promover la estabilidad y efectividad económicas que aseguren el pago de préstamos.

Las medidas de austeridad están diseñadas para estabilizar los niveles de deuda de un país y son una condición para acceder a mayor financiamiento del FMI. Sin embargo, si se las impone apenas superada una crisis económica, este tipo de

\footnotetext{
Protection". Disponible en inglés en: https://www.iilj.org/wp-content/uploads/2018/06/TheIMF-and-Social-Protection-NYU-IO-Clinic-Fall-2017.pdf.

41 Confederación Internacional de Organizaciones Sindicales Libres, "The IMF's Renewed Supply-Side Push: Four Decades of Structural Adjustment and Austerity Conditionality", (2020). Disponible en inglés en: https://www.ituc-csi.org/IMG/pdf/imf_s_renewed_supplyside_push.pdf.

42 Isabel Ortiz, et al., "The Decade of Adjustment: A Review of Austerity Trends 2010-2020 in 187 Countries", Documento de trabajo sobre ESS Núm. 53, (2015), OIT.
} 
prescripciones puede tener consecuencias dramáticas en las personas que viven en la pobreza y en el crecimiento económico en general. ${ }^{43} \mathrm{El}$ anterior Relator Especial de las Naciones Unidas sobre la pobreza extrema y los derechos humanos, Philip Alston, mostró que la privatización de servicios públicos puede conllevar a que su uso quede destinado únicamente a quienes puedan pagarlos $\mathrm{y}$, por lo tanto, marginalizar a quienes viven en la pobreza. ${ }^{44}$ Más aun, la decisión del Fondo de priorizar la estabilidad económica al promover la privatización de empresas públicas estatales y los recortes en el gasto social han conducido a impactos negativos sobre los sistemas de protección social.

En los últimos años, el FMI ha admitido que algunas respuestas menos radicales que las privatizaciones, como son la incorporación de mejoras en la administración de empresas públicas estatales, podría producir ganancias para el sector público, ${ }^{45}$ que podrían luego invertirse en promover intervenciones para la protección social. Recientemente, el Fondo también ha declarado encontrarse en un alejamiento de sus políticas de condicionalidades y, de esta manera, reconoce la importancia del gasto anticíclico, los costos de la desigualdad de ingresos y las consecuencias adversas de políticas inadecuadas en materia de protección social. ${ }^{46}$

Sin embargo, el FMI no ha cumplido su promesa de distanciarse del principio mismo de imponer condicionalidades $\mathrm{y}$, de

43 Informe del Relator Especial de las Naciones Unidas sobre la pobreza extrema y los derechos humanos Olivier De Schutter, “Looking Back to Look Ahead: A Rights-based Approach to Social Protection in the Post-COVID-19 Economic Recovery", (2020). Disponible en inglés en: https://www.ohchr.org/Documents/Issues/Poverty/Covid19.pdf.

44 Informe del Relator Especial de las Naciones Unidas sobre la pobreza extrema y los derechos humanos Philip Alston, "Privatización", (2018). Disponible en español y otros idiomas en: https://undocs.org/A/73/396.

45 FMI, "Managing Public Wealth", (2018), en: Monitor Fiscal. Disponible en inglés en: https://www.imf.org/en/Publications/FM/Issues/2018/10/04/fiscal-monitor-october-2018

46 Alexander E. Kentikelenis, et al., "IMF Conditionality and Development Policy Space, 1985-2014", en 4 Review of International Political Economy, 2016, vol. 23, 543-582. 
hecho, las ha incrementado. ${ }^{47}$ En concreto, la reducción de desembolsos de fondos públicos destinados a salud ha reforzado las desigualdades, y los índices de mortalidad neonatal han aumentado. Y si bien el Fondo agregó resguardos sociales a sus programas, en la práctica, no implicaron gran diferencia. ${ }^{48}$ El Proyecto Bretton Woods, una ONG que reúne a las voces críticas con el Banco Mundial y el FMI, ha atacado la implementación de medidas de austeridad porque quedan "institucionalizadas como la nueva norma" 49 y se ha expresado en contra de los shocks de ajuste porque afectan más duramente a los países en vías de desarrollo y sus poblaciones. ${ }^{50}$ En efecto, a pesar de las críticas como esta, hasta hoy se registran condicionalidades vinculadas a la consolidación fiscal, incluso en acuerdos tan recientes como los de respuesta del FMI a la COVID-19. ${ }^{51}$

\section{El Fondo en su papel de facilitador o filtro (gatekeeping) de préstamos de terceros}

La tercera vía por la cual el Fondo ejerce influencia sobre las políticas de cada país -y así también sobre la protección

\footnotetext{
47 Alexander E. Kentikelenis, et al., "IMF conditionality and development policy space, 1985-2014", en 4 Review of International Political Economy, 2016, vol. 23, 543-582.

48 Timon Forster, Alexander Kentikelenis, Thomas Stubbs y Lawrence King, "Globalization and Health Equity: The Impact of Structural Adjustment Programs in Developing Countries", en Social Science \& Medicine, 2019. Citado en: Kevin P. Gallagher y Franco Maldonado Carlin, "The Role of IMF in the Fight Against COVID-19: The IMF COVID-19 Recovery Index", 2020. Disponible en inglés en: https://www.bu.edu/gdp/2020/09/15/the-role-of-imf-in-the-fightagainst-covid-19-the-imf-covid-19-response-index/

49 Proyecto Bretton Woods, "IMF and World Bank Complicit in 'Austerity as New Normal', Despite Availability of Alternatives", 2019. Disponible en inglés en: https://www. brettonwoodsproject.org/2019/12/imf-and-world-bank-complicit-in-austerity-as-newnormal-despite-availability-of-alternative
}

50 Isabel Ortiz y Matthew Cummins, "Austerity: The New Normal. A Renewed Washington Consensus 2010-24", Documento de trabajo, (2019). Disponible en inglés en:https://www. ituc-csi.org/IMG/pdf/austerity_the_new_normal_ortiz_cummins.pdf

51 Soren Ambrose, "In the Midst of the Pandemic, Why is the IMF Still Pushing Austerity on the Global South?", en OpenDemocracy, 2020. Disponible en inglés en: https://www.opendemocracy.net/en/oureconomy/midst-pandemic-why-imf-still-pushing-austerity-global-south/ 
social- es mediante su apoyo a terceros. El Fondo tiene una función auditora única sobre otros prestamistas internacionales como, por ejemplo, los bancos de desarrollo. La aprobación del Fondo frente a las condiciones macroeconómicas y fiscales de un país lo afectan indirectamente, pues el organismo influye en su reputación para recibir aportes de otras entidades prestamistas internacionales.

Se ha sugerido que el Fondo podría utilizar su papel único de facilitador de préstamos de terceros para crear más espacio fiscal a nivel nacional mediante recomendaciones para la redistribución del gasto público, el incremento de la recaudación impositiva, la eliminación de los flujos financieros ilícitos y la movilización de recursos nacionales para expandir la protección social y/o reestructurar o cancelar deudas. ${ }^{52}$ Un ejemplo de esto, sería el hecho de introducir una nueva asignación de Derechos Especiales de Giro (DEG), como lo solicitaran diferentes organizaciones de la sociedad civil, especialmente en el contexto de la crisis de la COVID-19. ${ }^{53}$ No obstante, el Fondo ha mostrado resistencia a ir en estas direcciones y no está utilizando su postura única para promover financiamiento internacional en pos de reducir la pobreza y la desigualdad. ${ }^{54}$

\section{Respuesta del FMI a la crisis de la COVID-19}

La respuesta del Fondo a la crisis de la COVID-19 proporciona un terreno útil para poner a prueba en qué medida el organismo tiene capacidad de fortalecer la protección social o,

\footnotetext{
52 Isabel Ortiz y Matthew Cummins, "Austerity: The New Normal. A Renewed Washington Consensus 2010-24", Documento de trabajo, (2019), 50-51. Disponible en inglés en: https:// www.ituc-csi.org/IMG/pdf/austerity_the_new_normal_ortiz_cummins.pdf

53 Globalcovidresponse.org, "A Global COVID-19 Response with Special Drawing Rights", (2020). Disponible en inglés en: https://globalcovidresponse.org/

54 Clínica sobre organizaciones internacionales en la Facultad de Derecho de la Universidad de Nueva York (International Organizations Clinic at NYU School of Law) (2018), "IMF and Social Protection", Disponible en inglés en: https://www.iilj.org/wp-content/uploads/2018/06/ The-IMF-and-Social-Protection-NYU-IO-Clinic-Fall-2017.pdf
} 
por el contrario, de menoscabar los esfuerzos de un país en pos de ella. El análisis de dicha respuesta ilustra el marcado contraste entre los esfuerzos en pos de responder a las necesidades de financiamiento de un país y la satisfacción de condicionalidades impuestas en nombre de la sustentabilidad fiscal.

\section{Disponibilidad de financiamiento aunque en montos insustanciales}

Para los países que ya se encontraban en vías de desarrollo y situación vulnerable, la crisis supuso un grado de exposición aún mayor y los condujo a shocks económicos, lo cual evidencia aún más su necesidad de disponer de liquidez rápidamente para responder a las crisis. La ONU y el FMI han estimado que el mercado emergente y los países en desarrollo "necesitarían al menos unos 2500 billones de dólares en 2020 y todavía más en 2021 para evitar el colapso económico". 55

El FMI tiene un papel importante a la hora de mejorar la capacidad de esos países para potenciar su liquidez. Sin embargo, su respuesta ha sido en realidad muy limitada respecto de las necesidades de estos países en vías de desarrollo durante la actual crisis. ${ }^{56}$ El financiamiento más reciente que el FMI y los bancos regionales pusieron a disposición para la crisis de la COVID-19 alcanza solamente unos 90110 millones de dólares, lo cual representa apenas un 12,6 \% de sus recursos disponibles. ${ }^{57}$ Del monto mencionado, 88000 millones de dólares representan la

\footnotetext{
55 Conferencia de las Naciones Unidas sobre Comercio y Desarrollo, "From the Great Lockdown to the Great Meltdown: Developing Country Debt in the Time of Covid-19", (2020). Disponible en inglés en: https://unctad.org/system/files/official-document/gdsinf2020d3_en.pdf; y también FMI (2020), Ronda de prensa de Kristalina Georgieva, el 27 de marzo de 2020. Disponible en inglés en: https://www.imf.org/en/News/Articles/2020/03/27/ tr032720-transcript-press-briefing-kristalina-georgieva-following-imfc-conference-call

56 Thomas Stubbs, et al., "Whatever it Takes? The Global Financial Safety Net, COVID-19, and Developing countries", en World Development, 2020, vol. 137.

57 Thomas Stubbs, et al., "Whatever it Takes? The Global Financial Safety Net, COVID-19, and Developing countries", en: World Development, 2020, vol. 137, 1.
} 
suma aprobada por el FMI para requerimientos de financiamiento de 84 países desde marzo de 2020: otra suma bastante trivial si se la compara con la brecha mencionada de 2500 billones de financiamiento. ${ }^{58} \mathrm{~A}$ esto se suma que de los 88000 millones, solo 36 se habían desembolsado hasta octubre de 2020.59

El FMI ofrece diferentes facilidades de crédito mediante las cuales los países pueden acceder a financiamiento, la mayoría de base concesional. Desde comienzos de marzo de 2020, el FMI ha expandido las facilidades para permitir que los países respondan a la pandemia. Las medidas adoptadas en ese sentido son, entre otras, el aumento o incluso la suspensión de límites de acceso a ciertos créditos, la expansión de fondos fiduciarios para permitir el alivio de deudas, o hasta la introducción de un instrumento nuevo, la llamada "línea de liquidez de corto plazo", que ofrece liquidez rápida para países miembros con "entramados de políticas públicas fuertes" ${ }^{\circ 0}$. Sin embargo, aun si todas estas medidas incrementan el acceso al crédito, no aumentan el monto total de dinero que el FMI deja disponible para responder a la crisis: "el único financiamiento realmente nuevo son los 285 millones de dólares comprometidos en el Fideicomiso de Contención y Alivio de Catástrofes, apenas una pequeña parte de la potencia prestamista que existe, y todavía muy lejos de los 2500 billones que se reconocen como necesarios ${ }^{61 "}$. A su vez, estos fondos de crédito solo pueden utilizarse como alivio de deuda y, como no son subvenciones, tendrán que ser devueltos al FMI.

\footnotetext{
58 Thomas Stubbs, et al., "Whatever it Takes? The Global Financial Safety Net, COVID-19, and Developing countries", en: World Development, 2020, vol. 137, 4.

59 Thomas Stubbs, et al., "Whatever it Takes? The Global Financial Safety Net, COVID-19, and Developing countries", en: World Development, 2020, vol. 137, 4.

60 FMI, "Short-term Liquidity Line (SLL)" Ficha de datos, (2020). Disponible en inglés en: https://www.imf.org/en/About/Factsheets/Sheets/2020/04/17/short-termliquidity-line\#: : text=As \%20demand \%20for \%20liquidity \%20has, spilling \%20over \%20 to \%20other \%20countries; Thomas Stubbs, et al., "Whatever it Takes? The Global Financial Safety Net, COVID-19, and Developing Countries", en: World Development, 2020, vol. 137.

61 Thomas Stubbs, et al., "Whatever it Takes? The Global Financial Safety Net, COVID-19, and Developing Countries", en: World Developmen, 2020, vol. 137, 2.
} 


\section{Renovadas condicionalidades y medidas de austeridad}

Aunque en muchos de los programas de financiamiento recientes el FMI no ha incluido condicionalidades en forma de medidas de austeridad ${ }^{62}$, persisten dudas acerca de la implementación de estos programas en la práctica. En octubre de 2020, a través de un análisis de ochenta países, Eurodad encontró que el Fondo en realidad está incrementando la cantidad de medidas de austeridad en sus programas otorgados más recientemente. Estas medidas serán implementadas hacia 2023 y equivalen a un promedio de casi $4 \%$ del PIB de estos países ${ }^{63}$. Más aún, en la mayoría de ellos, los planes de consolidación fiscal son en realidad hasta cinco veces superiores que los paquetes de respuesta de la COVID-19 implementados por los mismos países ${ }^{64}$.

El aumento de las medidas de austeridad, las recomendaciones de aumentar los recortes al gasto público y el incremento en la carga de deuda son tres factores que, tomados en conjunto, desplazan el costo de la crisis a los sectores más vulnerables. En Ecuador, por ejemplo, el recorte del gasto público y el impacto económico de la crisis han conducido al colapso del sistema de salud. Para obtener la reestructuración de la deuda con el FMI, Ecuador necesita responder con medidas de austeridad que redundarán en aproximadamente $6 \%$ de su PIB hacia $2023^{65}$. Ecuador es uno de varios países en vías de desarrollo que se enfrentan a las medidas de austeridad impuestas por el FMI. Eurodad muestra que en cuarenta de los ochenta países analizados se espera que se efectúen recortes del gasto por montos

62 Kevin P. Gallagher, "The IMF's Return to Austerity?", 2020. Disponible en inglés en: https://www.ips-journal.eu/topics/democracy/the-imf-held-hostage-4710/

63 Daniel Munevar, “Arrested Development. International Monetary Fund Lending and Austerity Post COVID-19", en Eurodad, 2020, 10.

64 Daniel Munevar, “Arrested Development. International Monetary Fund Lending and Austerity Post COVID-19", en: Eurodad, 2020, 11.

65 Daniel Munevar, "Arrested Development. International Monetary Fund Lending and Austerity Post COVID-19", en: Eurodad, 2020, 11. 
al menos equivalentes a su presupuesto de salud total ${ }^{66}$. Estos recortes influyen directamente en el establecimiento de sistemas efectivos de protección social y obstaculizan significativamente los esfuerzos en pos de una cobertura universal de salud.

Lo que más necesitan las economías emergentes y los países en vías de desarrollo para afrontar la crisis actual, además de liquidez, es el alivio de su deuda ${ }^{67}$. Después de la pandemia, muchos países quedarán en situación aún más vulnerable y se enfrentarán con una carga de la deuda aún mayor. Como lo muestra el estudio de Eurodad, treinta países tendrán una carga de deuda al menos equivalente a sus paquetes de respuesta a la COVID-19 hacia 2023. Con todo, la evaluación de sustentabilidad de deudas con el FMI clasifica estas dinámicas como "sustentables"68. Una solución basada en alivios de deuda podría permitirles dar respuestas a los países, mediante políticas necesarias para mitigar los efectos socio-económicos de la pandemia sin dejar atrás a los grupos poblacionales vulnerables ${ }^{69}$.

\section{Movilizar ganancias por medio de impuestos indirectos no es suficiente}

Existe evidencia creciente de que el traslado de beneficios de las corporaciones internacionales y la lucha contra los paraísos fiscales podría contribuir a la movilización de recursos a nivel interno y, por lo tanto, asistir en el abordaje del déficit público. Aun así, el FMI sigue apoyando el incremento

\footnotetext{
66 Daniel Munevar, "Arrested Development. International Monetary Fund Lending and Austerity Post COVID-19", en: Eurodad, 2020, 2.

67 Kevin P. Gallagher, "The IMF's Return to Austerity?", 2020. Disponible en inglés en: https://www.ips-journal.eu/topics/democracy/the-imf-held-hostage-4710/

68 Daniel Munevar, "Arrested Development. International Monetary Fund Lending and Austerity Post COVID-19", en: Eurodad, 2020; para consultar una lista más reciente y exhaustiva de los países de ingreso bajo (PIB o LIC, según su sigla en inglés), de septiembre de 2020, consultar (en inglés): https://www.imf.org/external/pubs/ft/dsa/dsalist.pdf

69 Thomas Stubbs, et al. (2020), "Whatever it Takes? The Global Financial Safety Net, COVID-19, and Developing Countries", en: World Development, Vol.137.
} 
de impuestos indirectos, como los aplicados al consumo o el IVA, sin importar el impacto desproporcionado que tiene en los sectores vulnerables de la población. Diversas investigaciones han mostrado que los impuestos indirectos no solo desplazan la carga impositiva a las poblaciones locales, los cual les dificulta una recuperación equitativa y en principio consolida las desigualdades existentes ${ }^{70}$, sino que además carecen de universalidad en países con amplios sectores de economía informal ${ }^{71}$.

\section{Conclusión}

El FMI posee un enfoque ambiguo en cuanto la protección social. Después de recibir numerosas críticas por la implementación de medidas de austeridad en países miembros, el organismo ha dado un cambio en su política que resulta prometedor, ya que promueve una nueva mirada sobre la protección social. Algunas promesas, como la reducción o incluso eliminación de condicionalidades y la promoción del gasto social, especialmente en cuestiones de salud, están presentes en su respuesta inicial a la COVID-19 ${ }^{72}$. Pero el Fondo no ha abandonado su foco principal en la estabilidad económica a través de medidas de austeridad. Persisten discrepancias entre los anuncios del FMI de a dar respuesta a la COVID-19 y los desembolsos de fondos concretos para que los países puedan responder a esta crisis.

El FMI debe adoptar un enfoque en cuanto a la protección social basado en los derechos humanos, que trascienda la idea de selectividad (targeting) en el destino de fondos. No solo

\footnotetext{
70 Munevar, Daniel (2020), “Arrested Development. International Monetary Fund Lending and Austerity Post COVID-19", en: Eurodad

71 Bernhard Reinsberg et al. (2020), "Taxing the People, Not Trade: the International Monetary Fund and the Structure of Taxation in Developing Countries", en 55 Studies in Comparative International Development, 278-304, 299-300.

72 FMI, "World Economic Outlook 2020", (2020), Disponible en inglés y español en: https://www.imf.org/en/Publications/WEO/Issues/2020/09/30/world-economic-outlookoctober-2020
} 
debe buscar mitigar los efectos de sus programas hacia los más vulnerables sino que debe promover pisos de protección social que sean compatibles con la sustentabilidad fiscal para permitir que los países creen suficiente espacio fiscal para la protección social $^{73}$. El FMI también debe mejorar su colaboración con otras organizaciones internacionales para lograr un sistema financiero internacional confiable frente a los desafíos socioeconómicos persistentes exacerbados por la pandemia de la COVID-19 y la posibilidad de futuros shocks. De hecho, si bien el Fondo a menudo se ha referido a la experiencia del Banco Mundial y se ha apoyado en ella, a la hora de abordar cuestiones de protección social, su cooperación con otras organizaciones sociales en estos temas ha sido siempre muy limitada ${ }^{74}$. Por ejemplo, la participación del FMI en el SPIAC-B no ha sido demasiado activa. De un total de once reuniones entre 2012 y 2019, el Fondo solo ha estado presente en cinco $^{75}$ y solo desde 2018 con personal de los estratos más altos. ${ }^{76}$ Dado el renovado interés

73 Informe del Relator Especial sobre la extrema pobreza y los derechos humanos Philip Alston, "The International Monetary Fund and its Impact on Social Protection", párrafo 56, 2018. Disponible en inglés en: https://srpoverty.org/wp-content/uploads/2018/08/internationalmonetary-fund-impact-on-social-protection-2018.pdf

74 Coalición Global por los Pisos de Protección Social, "The IMF's New Policy Framework on Social Protection", (2019). Disponible en inglés en: http://www.socialprotectionfloorscoalition.org/2019/01/the-imfs-new-policy-framework-on-social-protection/; Clínica sobre organizaciones internacionales en la Facultad de Derecho de la Universidad de Nueva York (International Organizations Clinic at NYU School of Law) (2018), "IMF and Social Protection", disponible en inglés en: https://www.iilj.org/wp-content/uploads/2018/06/The-IMF-and-Social-Protection-NYU-IO-Clinic-Fall-2017.pdf Oficina de Evaluación Independiente del Fondo Monetario Internacional, "The IMF and Social Protection", Informe de evaluación (2017). Disponible en inglés en: https://ieo.imf.org/en/our-work/Evaluations/Completed/20170724-the-imf-and-social-protection.

75 OIT, Social Protection Inter-agency Cooperation Board, Consejo de Cooperación Interinstitucional en Materia de Protección Social. (SPIAC, por su sigla en inglés) Reuniones del SPIAC-B. Documentos disponibles en inglés en: https://www.ilo.org/newyork/at-the-un/ social-protection-inter-agency-cooperation-board/lang--en/index.htm.

76 FMI, Documento Normativo (IMF Policy Paper) Núm. 20/012, (2020): https://www.imf. org/en/Publications/Policy-Papers/Issues/2020/03/04/Tenth-Periodic-Monitoring-ReportOn-The-Status-Of-Management-Implementation-Plans-In-49245. 
por la protección social, el FMI debe ampliar los mecanismos de cooperación con otras organizaciones internacionales con referencia a este tema, lo cual involucra al Banco Mundial, desde ya, pero también a UNICEF y la OIT. ${ }^{77}$ La importancia de esta cooperación también ha sido resaltada por el propio FMI en el ya mencionado informe de septiembre de $2020 .{ }^{78}$ Una acción clara que puede contribuir a la evolución del enfoque del FMI sobre la protección social sería su participación en las conversaciones para el establecimiento de un Fondo Global para la Protección Social para Todos, iniciativa que promueven el gobierno francés y el Relator especial de las Naciones Unidas sobre la extrema pobreza y los derechos humanos.

77 Oficina de Evaluación Independiente del Fondo Monetario Internacional, Informe de evaluación, "The IMF and Social Protection", (2017). Disponible en inglés en: https://ieo.imf. org/en/our-work/Evaluations/Completed/2017-0724-the-imf-and-social-protection.

78 Irene Yackovlev, et al., "How to Operationalize IMF Engagement on Social Spending during and in the aftermath of the COVID-19 Crisis", en How To Note 20/02 FMI, 14 de septiembre de 2020, 2. Disponible en inglés en: https://www.imf.org/en/ Publications/Fiscal-Affairs-Department-How-To-Notes/Issues/2020/09/14/How-to-Operationalize-IMF-Engagement-on-Social-Spending-during-and-in-the-aftermath-of-the-49718 